\title{
Impact of wearing a facial covering on aerobic exercise capacity in the COVID-19 era: is it more than a feeling?
}

\author{
Matthew Kampert ${ }^{1} \cdot$ Tamanna Singh $^{1}$ - J. Emanuel Finet ${ }^{2} \cdot$ Erik H. Van Iterson $^{3}$ (1)
}

Received: 24 July 2020 / Accepted: 30 July 2020 / Published online: 24 August 2020

(c) Springer-Verlag GmbH Germany, part of Springer Nature 2020

Keywords Severe acute respiratory syndrome coronavirus $2 \cdot$ SARS-CoV-2 2 Exercise intolerance $\cdot$ CPET $\cdot$ Facial mask

Sirs:

In this issue of Clinical Research in Cardiology, Drs. Fikenzer and colleagues [1] should be acknowledged for their timely study focused on examining how 'cardiopulmonary exercise capacity' may be impacted by wearing a nose and mouth facial covering during cardiopulmonary exercise testing (CPET). They are the first to report in a randomized-controlled cross-over study design that when CPET is performed by healthy young-to-middle aged adult men while wearing an FFP2/N95 mask (ffpm), and to a lesser extent with a surgical mask (sm), key exercise measurements, including peak oxygen uptake $\left(V \mathrm{O}_{2 \text { peak }}\right)$, maximum Power (Pmax), and peak minute ventilation $\left(V_{E}\right)$ do not increase to levels demonstrated during CPET with no mask (nm) [1]. When such differences are taken together with additional data acquired at rest suggesting pulmonary function testing while wearing an ffpm also yields significantly decreased lung function as

This comment refers to the article available online at https://doi. org/10.1007/s00392-020-01704-y.

Erik H. Van Iterson

vanitee@ccf.org

1 Section of Clinical Cardiology, Robert and Suzanne Tomsich Department of Cardiovascular Medicine, Miller Family Heart, Vascular and Thoracic Institute, Cleveland Clinic, 9500 Euclid Ave., Desk J2-4, Cleveland, OH 44195, USA

2 Section of Heart Failure and Transplantation Medicine, Robert and Suzanne Tomsich Department of Cardiovascular Medicine, Miller Family Heart, Vascular and Thoracic Institute, Cleveland Clinic, 9500 Euclid Ave., Desk J3-4, Cleveland, OH 44195, USA

3 Section of Preventive Cardiology and Rehabilitation, Robert and Suzanne Tomsich Department of Cardiovascular Medicine, Miller Family Heart, Vascular and Thoracic Institute, Cleveland Clinic, 9500 Euclid Ave., Desk JB-1, Cleveland, OH 44195, USA compared with the condition of nm, the authors emphasize that wearing an ffpm (or even an sm) during CPET imposes appreciable limitations on cardiopulmonary function when exercise is performed at high intensity. The authors conclude with the bold recommendation for readers regarding the wearing of ffpm or sm coverings during exercise participation, "These effects have to be considered versus the potential protective effects of face masks on viral transmissions. The quantitative data of this study may, therefore, inform medical recommendations and policy makers [1]." Thus, because there are clear clinical implications associated with implementing and closely adhering to expert-guided safety precautions aimed at helping to minimize public transmission of the novel coronavirus disease 2019 (COVID-19) [2], we felt that it would be instructive and responsible to communicate our concerns over the interpretation of quantitative and qualitative data acquired during CPET performed while wearing $\mathrm{nm}$ as compared to CPET performed with an ffpm or sm as discussed by Fikenzer et al. [1].

An initial focus of our concern is that Fikenzer et al. [1] offer support for their interpretation of quantitative data based on the physiological concept [3] that wearing an ffpm/ sm during CPET markedly increases breathing resistance to yield exercise limiting elevations in work of breathing, altered ventilatory mechanics, cardiac hemodynamic compensation, and disproportionately increased cardiac output redistributed to respiratory muscles. While this is a plausible cascade of events provoked by wearing an $\mathrm{ffpm} / \mathrm{sm}$ during CPET, it is unclear on what physiological basis they arrived at such a causal pathway for their study, since (1) they did not perform measurements of pulmonary function, work of breathing, and ventilatory mechanics during CPET, (2) actual data reported are incompletely listed, and (3) the fundamental model of blood flow redistribution at peak exercise described by Harms et al. [3] is not properly translated and applied. 
Harms et al. [3] demonstrate that increasing exercise inspiratory muscle work of breathing by $20-80 \%$ above control exercise levels does not result in significant between condition differences in peak cardiac output, stroke volume, arterial-mixed venous $\mathrm{O}_{2}$ difference, $V_{E}$, respiratory rate, tidal volume, and $V \mathrm{O}_{2 \text { peak }}$. By contrast, it is the result of exercise inspiratory muscle unloading where Harms et al. [3] reports significantly decreased peak cardiac output and $V \mathrm{O}_{2 \text { peak }}$ as compared to control exercise. However, none of the CPET conditions reported in Fikenzer et al. [1] included inspiratory muscle unloading and it is inappropriate to consider CPET performed with $\mathrm{nm}$ as an inspiratory unloaded condition. Moreover, neither ffpm nor sm wearing should be considered interventions leading to inspiratory muscle unloading. The misinterpretation of exercise physiological mechanisms originally reported in Harms et al. [3] is not a trivial oversight made by Fikenzer et al. [1], since the basis of their study conclusion is formulated on the misguided notion that their data and study design suggest increasing breathing resistance by wearing an ffpm/sm in healthy adult men causes physiological limitations to explain reductions in aerobic exercise capacity. Extrapolation of pulmonary and exercise data acquired in patients with chronic obstructive pulmonary disease wearing a N95 mask while performing the 6 min walk test also provides no relevant evidence to support views expressed by the authors $[1,4]$.

Another concern stems from the fact that exercise physiological comparisons discussed in Fikenzer et al. [1] are not formulated on the basis of comparable levels of "peak" exercise metabolic demand. Although the authors do not report peak exercise respiratory exchange ratio for each CPET condition, owing to the significant group effect for peak exercise lactate coupled with proportional reductions in $V \mathrm{O}_{2 \text { peak }}$, Pmax, $V_{E}$, respiratory rate, and tidal volume listed in their results [1], it is likely that participants did not terminate CPET at equivalent levels of metabolic demand across conditions. The authors did not properly take into account the considerably higher peak metabolic demand incurred during CPET with $\mathrm{nm}$ as compared to CPET with an ffpm $(12.8 \pm 3.09$ vs. $10.8 \pm 3.12$ lactate, respectively) when discussing whether $V \mathrm{O}_{2 \text { peak }}$, Pmax, and $V_{E}$ actually differed between study conditions. The lack of statistical significance for pairwise comparisons of peak lactate between CPET with $\mathrm{nm}$ as compared to the ffpm condition likely reflects an underpowered study as evidenced by a very large estimated effect size exceeding 1.40 calculated herein.

In contrast to the strong attention that authors afforded to stress the relevance of quantitative differences in CPET responses, the overwhelming negative impact that wearing an ffpm had on the perceived discomfort of CPET received modest secondary consideration. Both the overall and individual scoring for each domain of perceived discomfort associated with wearing an ffpm during CPET contradicts the suggestion that, "the data suggest the associated discomfort as a second important reason for the observed impairment of physical performance." As an example, the mean score for perceived discomfort of breathing resistance reached $7.4 \pm 2.5$ for CPET with an ffpm (0-10 scale; 10 is maximal discomfort), amounting to more than four times greater severity of discomfort as compared to CPET with $\mathrm{nm}$. Thus, without exercise physiological evidence demonstrating participants performed cross-over CPET bouts at comparable levels of metabolic demand, the authors are not at liberty to discount the overwhelmingly negative perceived discomfort of performing CPET with an ffpm as a primary cause for exercise termination.

Our concerns expressed herein underscore that exercise participation in any public setting should not at this time be excluded from current expert-guided [2] universal recommendations strongly encouraging the public display of nose and mouth facial coverings to help curb the transmission of COVID-19.

Funding None.

\section{Compliance with ethical standards}

Conflict of interest The authors report no conflicts of interest.

Ethical approval Not applicable.

\section{References}

1. Fikenzer S, Uhe T, Lavall D et al. (2020) Effects of surgical and FFP2/N95 face masks on cardiopulmonary exercise capacity. Clin Res Cardiol 1-9. https://link.springer.com/article/10.1007/s0039 2-020-01704-y. Accessed 10 July 2020

2. Centers for Disease Control and Prevention (2020) Coronavirus Disease 2019 (COVID-19), How to Protect Yourself \& Others. https://www.cdc.gov/coronavirus/2019-ncov/prevent-gettingsick/prevention.html?CDC_AA_refVal=https $\% 3 \mathrm{~A} \% 2 \mathrm{~F} \% 2 \mathrm{Fwww}$ .cdc.gov\%2Fcoronavirus\%2F2019-ncov\%2Fprepare\%2Fpreventi on.html. Accessed 07/24/2020

3. Harms CA, Wetter TJ, McClaran SR et al (1998) Effects of respiratory muscle work on cardiac output and its distribution during maximal exercise. J Appl Physiol (1985) 85(2):609-618

4. Kyung SY, Kim Y, Hwang H, Park JW, Jeong SH (2020) Risks of N95 face mask use in subjects with COPD. Respir Care 65(5):658-664 